\title{
TAKOTSUBO CARDIOMYOPATHY ASSOCIATED WITH PERIMYOCARDITIS: YET ANOTHER IMPORTANT DIFFERENTIAL DIAGNOSIS TO ENTERTAIN
}

Singapore Med J 2015; 56(5): 304-305 doi: 10.11622/smedj.2015083

Dear Sir,

We read with great interest the report by Lee et al, ${ }_{(1)}^{(1)}$ who did a commendable job of illustrating the clinical pearls in perimyocarditis and emphasised the importance of recognising its electrocardiographic evolution/staging. However, we would like to point out an additional important diagnostic electrocardiographic marker.

In the first electrocardiogram (ECG) of Lee et al's report, ${ }^{(1)}$ downsloping of TP segments, known as Spodick's sign, ${ }^{(2)}$ which is named after the pioneer work of our former university professor Dr David Spodick, is observed. This finding is best noted in lead II and the lateral precordial leads, and is present in about $80 \%$ of cases of acute pericarditis (Fig. 1). It also serves as an excellent electrocardiographic tool for differentiating acute pericarditis from acute coronary syndrome.

The electrocardiographic pattern observed in the second ECG, viz. convex tomb-stoning ST elevations with absence of PR segment depression, is unusual for the electrocardiographic evolution of pericarditis or perimyocarditis, though it may be observed (albeit rarely) in frank myocarditis or myopericarditis. ${ }^{(3)}$ Since the diagnosis was based mostly on the clinical context, we wish to emphasise the importance of entertaining an alternate differential diagnosis or clinical explanation.

The understanding of the pathophysiology and risk factors for takotsubo cardiomyopathy (TC) has been a continuously evolving process. The association between classical- or variant-form (regional) TC and perimyocarditis has been recognised recently and emphasised in one of our prior works. ${ }^{(4)}$ The preceding myopericarditis can thus serve as a stressor substrate for TC.

Thus, we wonder if Lee et $\mathrm{al}^{\prime} \mathrm{s}^{(1)}$ finding from the second ECG can be better explained by TC or diffuse epicardial coronary vasospasm (one of the potential pathophysiological mechanisms for TC). The electrocardiographic evolution of TC is very similar to the four-stage evolution of pericarditis. ${ }^{(5)}$ Furthermore, in Lee et al's report, ${ }^{(1)}$ the presence of apical hypokinesis, in addition to a midinferoseptal hypokinesia, may highly support our hypothesis of atypical TC superimposed on the preceding perimyocarditis. Did the authors perform a repeat echocardiography to observe any improvement in wall function? An early resolution of such hypokinesis may again be supportive of TC. Nevertheless, cardiac magnetic resonance imaging may be particularly useful for making the final diagnosis in such cases. ${ }^{(6,7)}$ The telltale sign (i.e. the presence of epicardial and mid-wall late gadolinium enhancement, and an enhanced focal T2 signal representing myocardial oedema) is diagnostic of perimyocarditis. ${ }^{(8)}$ On the other hand, the lack of apical late gadolinium enhancement will be more supportive of superimposed TC.

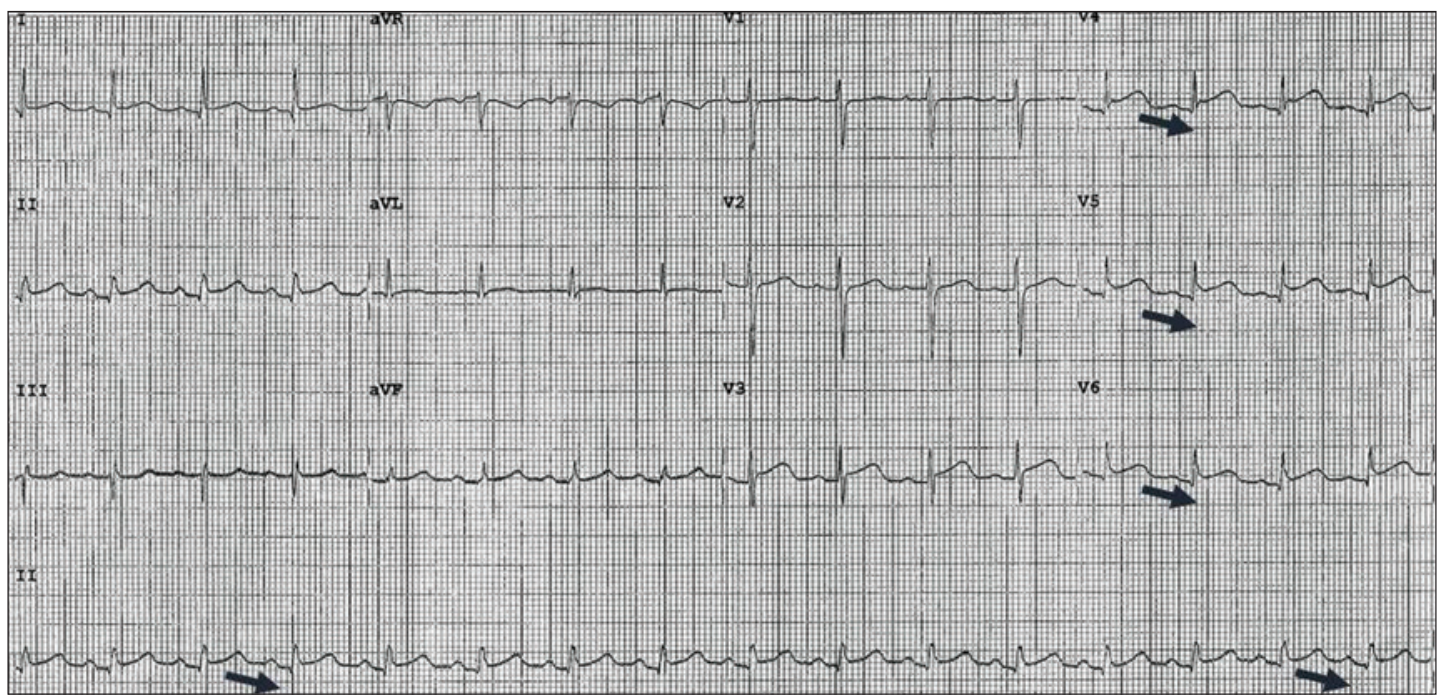

Fig. 1 Initial ECG (from Lee et al's case report ${ }^{(1)}$ ) shows Spodick's sign, which is the downsloping TP segments in lateral precordial leads, viz. V4, V6, and lead II (black arrows [inserted by current authors]).

Yours sincerely,

Lovely $\underline{\text { Chhabra }}^{1}$, Vinod $\mathrm{K}$ Chaubey $^{2}$

${ }^{1}$ Department of Cardiovascular Medicine, Hartford Hospital, University of Connecticut School of Medicine, Hartford, CT, ${ }^{2}$ Department of Medicine, Saint Vincent Hospital, Worcester, MA, USA. azygus@gmail.com

\section{REFERENCES}

1. Lee PT, See CK, Chiam PT, Lim ST. Electrocardiographic changes in acute perimyocarditis. Singapore Med J 2015; 56:e1-3.

2. Chaubey VK, Chhabra L. Spodick's sign: a helpful electrocardiographic clue to the diagnosis of acute pericarditis. Perm J $2014 ; 18:$ e122.

3. Chhabra L. Is positive troponin a hype in myopericarditis: should emergency room risk stratification criteria be revisited? J Cardiovasc Med (Hagerstown) 2015; 16:143.

4. Chhabra L, Khalid N, Kluger J, Spodick DH. Lupus myopericarditis as a preceding stressor for takotsubo cardiomyopathy. Proc (Bayl Univ Med Cent) 2014; 27:327-30 
5. Zhong-qun Z, Chong-quan W, Sclarovsky S, et al. ST-segment deviation pattern of takotsubo cardiomyopathy similar to acute pericarditis: diffuse ST-segment elevation. J Electrocardiol 2013; 46:84-9.

6. Friedrich MG, Strohm O, Schulz-Menger J, et al. Contrast media-enhanced magnetic resonance imaging visualizes myocardial changes in the course of viral myocarditis. Circulation 1998; 97:1802-9.

7. Chaubey VK, Chhabra L, Kaur NJ, Spodick DH. Regional pericardits: a mischievous masquerader. North Am J Med Sci 2015; 7:32.

8. Chhabra L. Myopericarditis and Takotsubo cardiomyopathy association. Int J Cardiol. 2015; 186:143. 